\title{
Factores de riesgo maternos asociados a retinopatía del prematuro
}

\section{Maternal risk factors associated with retinopathy of Premature}

Recibido: 22/06/2021 Aceptado: 26/07/2021

\author{
Nulvi Stefanni Portillo Sosa \\ Universidad San Carlos de Guatemala \\ stefannisosa3@gmail.com \\ https://orcid.org/0000-0001-5613-9878
}

\section{Referencia del artículo}

Portillo Sosa, N. S. (2021). Factores de riesgo maternos asociados a retinopatía del prematuro. Revista Diversidad Científica, 1(1), 35-44. DOI: https://doi.org/10.36314/diversidad.v1i1.5

\section{Resumen}

OBJETIVO: describir los factores de riesgo maternos asociados a retinopatía del prematuro (ROP). MÉTODO: se recopiló información en Pubmeed, Cochrane, revistas, libros, se encontraron metaanálisis, artículos y guías sobre ROP. RESULTADOS: la edad materna tuvo una relación estadística en estudios anteriores con un valor $\mathrm{P}:<0.04$, un estudio en Perú mostr $20.6 \%$ de los pacientes hijos de madres preclamticas desarrollo ROP, hijos de madres con diabetes mellitus (DM) también mostraron mayor incidencia, el tabaquismo aumento el VEGP/PEDF y por tanto mayor incidencia de ROP, el conteo elevado de glóbulos blancos tuvo un OR: 4.37 y un valor P:0.019 siendo significativo, en otro estudio un $26.4 \%$ de las mamas con anemia por deficiencia de hierro presentaron ROP. CONCLUSIÓN: se describe asociación estadística a ROP en los factores biológicos, obstétricos, patológicos y psicosociales. Se identifica a la edad materna como un factor de riesgo biológico indirecto; al tabaquismo como un factor de riesgo psicosocial; la hipertensión durante el embarazo, la DM, ITU, anemia por deficiencia de hierro y el aumento del recuento de glóbulos blancos como factores de riesgo patológicos; por último, a la edad gestacional, RTIU, RPMO, vía de resolución del parto, concepción asistida y corioamnionitis como factores de riesgo obstétricos.

Palabras clave: retinopatía del prematuro y factores de riesgo 


\section{Abstract}

TARGET: describe the maternal risk factors associated with retinopathy of prematurity (ROP). METHOD: information was collected in Pubmeed, Cochrane, journals, books, meta-analyzes, articles and guides on ROP were found.. RESULTS: maternal age had a statistical relationship in previous studies with a P value: $<0.04$, a study in Peru showed $20.6 \%$ of patients children of preeclamatic mothers developed ROP, children of mothers with diabetes mellitus (DM) also showed a higher incidence, smoking increased VEGP / PEDF and therefore a higher incidence of ROP, the elevated white blood cell count had an OR: 4.37 and a $P$ value: 0.019 being significant, in another study $26.4 \%$ of the breasts with anemia due to deficiency of iron presented ROP. CONCLUSION: it is described Statistical association to ROP in biological, obstetric, pathological and psychosocial factors. Maternal age is identified as an indirect biological risk factor; smoking as a psychosocial risk factor; hypertension during pregnancy, DM, UTI, iron deficiency anemia and increased white blood cell count as pathological risk factors; bylatest, at gestational age, IUGR, RPMO, delivery resolution pathway, assisted conception and chorioamnionitis as obstetric risk factors.

Keywords: retinopathy of prematurity and risk factors 


\section{Introducción}

La Retinopatía del prematuro es una vitreorretinopatía proliferativa periférica que ocurre por una alteración en la vasculogénesis ocasionando vasoconstricción y obliteración del lecho capilar, seguido de falla en la perfusión e hipoxia local, la cual induce a la formación de neovascularización, es decir tejido fibrovascular proliferativo extrarretiniano, que en etapas avanzadas se extiende al humor vítreo provocando hemorragias, exudación, tracción sobre la retina y por último desprendimiento de ésta, siendo la principal causa de ceguera infantil (Camba Longueira, et al., 2008).

Tiene una etiología multifactorial, siendo la inmadurez su principal factor de riesgo, el desarrollo de la red sanguínea del ojo comienza a las 16 semanas, desde la parte central posterior hacia la periferia, culminando su desarrollo a las 40 semanas en la retina nasal y a las 42 semanas en la retina temporal. Por consiguiente, cuando nace un prematuro la retina no ha completado aún su desarrollo y la periferia carece de vasos sanguíneos (Cauich, 2017).

La Organización Mundial de la Salud (2018) reporta que cada año 15 millones de bebés nacen de forma prematura (antes de las 37 semanas de gestación y con peso inferior de 2,000 gr) y mueren 1 millón de recién nacidos prematuros convirtiéndose en la segunda causa de mortalidad infantil en el mundo y en la principal causa de ceguera (OMS, 2018).

De toda la población, la infantil es un grupo prioritario en el manejo de los defectos visuales, puesto que enfrenta un proceso de aprendizaje, para los cuales dichas alteraciones no corregidas pueden implicar dificultades en el desempeño escolar y retraso en el proceso de desarrollo psicosocial (UNICEF, 2013).

La objetividad del estudio fue describir los factores de riesgo maternos asociados a retinopatía del prematuro, identificar los factores de riesgo biológicos maternos asociados a retinopatía del prematuro, identificar los factores de riesgo psicosociales maternos asociados a retinopatía del prematuro, identificar los factores de riesgo obstétricos maternos asociados a retinopatía del prematuro e identificar los factores de riesgo patológicos maternos asociados a retinopatía del prematuro.

Se identificó a la edad materna (mujeres en edades muy jóvenes o muy avanzadas) como un factor de riesgo biológico indirecto para ROP; al tabaquismo como un factor de riesgo psicosocial según metaanálisis de alta evidencia; la hipertensión durante el embarazo, la diabetes mellitus, infección del tracto urinario, anemia por 
deficiencia de hierro y el aumento del recuento de glóbulos blancos como factores de riesgo patológicos; por último a la edad gestacional, restricción del crecimiento intrauterino, ruptura de membranas, vía de resolución del parto, concepción asistida y corioamnionitis como factores de riesgo obstétricos.

\section{Contenido}

La retinopatía del prematuro (ROP) es un trastorno retiniano vasoproliferativo exclusivo de los bebés prematuros. El desarrollo vascular retiniano comienza durante la semana 16 de gestación. El tejido mesenquimatoso (la fuente de los vasos retinianos) crece de manera centrífuga desde el disco óptico, alcanzando la ora serrata nasal a las 36 - 40 semanas de gestación y la ora serrata temporal a las 40 - 42 semanas de gestación (Cauich, 2017).

La retinopatía del prematuro es una enfermedad vasoproliferativa de origen multifactorial producida por una retina inmadura y sólo parcialmente vascularizada al momento del nacimiento, se ah identificado varios factores de riesgo maternos que pueden llevar al desarrollo de ROP, dentro de los cuales se encuentran:

Yau y sus colaboradores en un estudio multivariado publicado en el año 2015 encontraron a la edad materna muy vieja o muy joven como un factor de riesgo para el desarrollo de retinopatía del prematuro ( $P \leq 0,04)$ (Yau, et al., 2015).

En Perú durante el año 2016, se publicó un estudio donde la población muestral fue de 136 recién nacidos prematuros, obteniendo como resultados: Infecciones del Tracto Urinario con un 44,1\%, 28,7\% con Preeclampsia otras hacen Eclampsia, el $20,6 \%$ con Hipertensión Arterial, el 19,9\% Anemia y por último $18,4 \%$ con Diabetes Mellitus (Guerrero Sausa, et al., 2016).

La diabetes puede tener un impacto directo (por ejemplo, aumento del factor de crecimiento endotelial vascular (VEGF) retiniano por hiperglucemia) como indirecto (por ejemplo, asociación con el síndrome de dificultad respiratoria) en el desarrollo de ROP. El Ensayo Nacional de Colaboración sobre el Ductus Arteriosus de Patentes en los Estados Unidos de 1979 a 1981 encontró una mayor incidencia de ROP entre los bebés de madres diabéticas. Recientemente, un estudio retrospectivo turco identificó la DM materna como un factor de riesgo independiente tanto para ROP como para ROP tipo 1 en bebés con BW $\geq 1500$ gr. (Kim, 2018). 
Un estudio realizado en 2015 publicado en Basilea se encontró que nornicotina y nicotina inducen neovascularización a través del aumento de la relación VEGF/ PEDF. Las influencias de la nornicotina y la nicotina (NT) en el humo del cigarrillo en la expresión del factor de crecimiento endotelial vascular (VEGF) y el factor derivado del epitelio pigmentario (PEDF) en las células del epitelio pigmentario retiniano y las células endoteliales de venas umbilicales humanas (HUVECs). Además, los comportamientos angiogénicos de las células endoteliales bajo tratamiento con nornicotina y NT se evaluaron mediante el uso de métodos in vitro. Las células ARPE-19 y los HUVECs fueron tratados con diferentes concentraciones de nornicotina o NT durante diferentes períodos de tiempo. La migración HUVECs y la formación de tubos se evaluaron mediante el uso del ensayo de arañazos y los modelos Matrigel. Las expresiones del gen VEGF y PEDF y la proteína en ambos tipos de células fueron examinadas por RT-PCR y Western blot en tiempo real. No hubo proliferación de células ARPE-19 después del tratamiento con varias concentraciones de nornicotina o NT. Por el contrario, la nornicotina o nicotina estimularon significativamente la proliferación, migración y formación de tubos de HUVEC. Nornicotina y NT regularon la expresión de VEGF (Zhang, 2015).

En un estudio realizado en Turquía, se observó que de 254 bebes $187(73,6 \%)$ presentaron deficiencia de hierro, mientras que los 67 restantes $(26,4 \%)$ no tenían deficiencia. Los bebés nacidos de madres con anemia por deficiencia de hierro con hemoglobina marcadamente disminuida, hematocrito, volumen corpuscular medio, hierro sérico y niveles de ferritinatina tenían más probabilidades de desarrollar retinopatía de prematuridad (Dai, 2015).

Existe un estudio de cohorte retrospectiva. La muestra a estudio fue de 246 bebés con edad gestacional $\leq 32$ semanas, con exámenes histológicos de sus placentas. ROP fue diagnosticado en 82 de 246 bebés (33,3\%), incluyendo 49 con ROP leve y 33 con ROP grave. El análisis de regresión multivariante indicó que la corioamnionitis clínica y el recuento de glóbulos blancos materno elevado cuentan con la admisión para estar asociado con la aparición de ROP [relación de probabilidades $(O R)=4.370, P=0,046$; y OR = 1,104 por 1.000 células $/ \mathrm{mm} 3$ aumento incremental, $P=0,019$, respectivamente], el recuento elevado de glóbulos blancos materno en la admisión también se asoció independientemente con la progresión ROP que requiere tratamiento con láser $(\mathrm{OR}=1.171$ por cada 1.000 células $/ \mathrm{mm} 3$ aumento incremental, $P=0,026)$ (Woo, 2012).

En un estudio prospectivo de cohortes realizado en Alemania, se realizó una encuesta de detección prospectiva de ROP que inscribió a todos los prematuros admitidos en la UCIN de enero de 2009 a diciembre de 2010, con una edad gestacional de 32 semanas o menos al nacer y un peso al nacer de $1500 \mathrm{~g}$ o menos. Un total de 
172 bebés ( 88 mujeres) se sometió a una evaluación de la retina por oftalmoscopia indirecta a partir de la semana posnatal $4\left({ }^{a}\right)$ y se les dio seguimiento periódicamente. Los factores de riesgo perinatal para ROP se evaluaron utilizando análisis univariados y multivariantes. Los bebés que avanzaron a la etapa 3 ROP con más enfermedad recibieron terapia láser. El análisis univariado mostró que había una relación significativa entre la aparición de ROP y la edad gestacional $(P=0,000)$, sepsis $(P=0,004)$, oxigenoterapia $(P=0,018)$ y frecuencia de transfusiones de sangre $(P=0,030)($ Abel, 2012).

La gestación múltiple se asocia con un mayor riesgo de parto prematuro, muy bajo peso y morbilidades perinatales, lo que puede afectar el riesgo de ROP. En el estudio CRYO-ROP, se encontró que los bebés únicos tenían menos riesgo de ROP que los gemelos o los múltiplos (Kim, 2018).

En un estudio de casos y controles en la ciudad de México la muestra estuvo constituida por 282 recién nacidos que, para fines de comparación, se dividieron en dos grupos: $152(53.9 \%)$ con retinopatía y $130(46.1 \%)$ sin la enfermedad. Se detectaron diferencias en la vía de resolución del embarazo por cesárea $(p=0.038)$ (Rivera-Rueda, et al., 2020).

Aunque el mecanismo exacto no está claro, la asociación entre la tecnología de reproducción asistida (TAR) incluida la fertilización in vitro (FIV) y el ROP se ha estudiado con resultados contradictorios. Algunos estudios identificaron el TAR como un factor de riesgo independiente para el ROP, pero es difícil sacar conclusiones definitivas porque el TAR también está asociado con factores como el bajo BW, los primeros GA y los partos múltiples. Dos estudios del Reino Unido sugieren que las mejoras en la FIV pueden haber reducido el riesgo adicional de ROP grave (Kim, 2018).

Inflamación intrauterina incluyendo corioamnionitis se ha asociado con varias morbilidades perinatales y varios estudios sugieren asociación de corioamnionitis con ROP. Un metaanálisis de 27 estudios en 2014 reveló que la corioamnionitis se asoció significativamente con ROP por análisis univariados, pero no se encontró ninguna asociación en el análisis multivariante que corregía para edad gestacional. Un estudio reciente sugirió una asociación entre ROP posterior agresivo (APROP) y corioamnionitis (Kim, 2018).

Los datos publicados son contradictorios sobre la asociación de la ruptura prematura de las membranas (PPROM) y el riesgo ROP. Un estudio turco de un solo 
centro encontró que PPROM >18 horas se asoció independientemente con un mayor riesgo de ROP tipo 1; sin embargo, un estudio sueco de control de casos coincidente en WINROP (peso, insulina-como factor de crecimiento-1, neonatal, ROP) sistema de alarma encontró PPROM para tener un efecto protector contra la etapa ROP $\geq 3$. Estos resultados contradictorios podrían explicarse por diferentes medidas de resultados, tamaño de muestra pequeño y diferentes factores de confusión ajustados en esos estudios. (Kim, 2018).

Datos recopilados en la Red Neonatal de Australia y Nueva Zelanda de neonatos de alto riesgo (peso al nacer de $<1500 \mathrm{~g}$ o edad gestacional [AG] de $<32$ semanas, fueron examinados para el subconjunto, niños con el GA de $<29$ semanas que sobrevivieron a las 36 semanas $(n=2105)$. La prematuridad fue el factor de riesgo dominante, con neonatos con AG de $<25$ semanas con 20 veces mayores probabilidades de ROP grave que los neonatos con AG de 28 semanas. El peso al nacer para la AG también tuvo un efecto de "dosis-respuesta"; los niños más restringidos al crecimiento tuvieron mayor riesgo, con los neonatos por debajo del percentil 3 de peso para la AG que tenían 4 veces más probabilidades de RP grave que aquellos entre los percentiles 25 y 75 . El sexo masculino también fue un factor de riesgo significativo (Odds ratio: 1,73; intervalo de confianza del 95\%: 1,25-2,40). Estos datos, para una cohorte grande, esencialmente sobre la base de la población, sugieren que los factores relacionados con el grado de inmadurez, la restricción del crecimiento intrauterino, y el género masculino contribuyen al ROP severo (Darlow, et al., 2005).

Un estudio que se realizó en el Hospital Regional Docente las Mercedes - Chiclayo, evaluó factores neonatales en 31 neonatos con diagnóstico de ROP y 62 controles. De los factores maternos los que más relevancia tuvieron fueron las Infecciones del Tracto Urinario con un $44,1 \%$, así mismo con un $28,7 \%$ con preeclampsia, otras hacen eclampsia, el $20,6 \%$ con hipertensión arterial, el 19,9\% anemia y por último $18.4 \%$ con diabetes mellitus. Según Rodríguez de los factores de riesgo las infecciones urinarias se encontraron en un $39 \%$. Zamora, en su estudio, encuentra que la complicación materna más frecuentes encontradas fueron algunas infecciones del aparato genital que se asocian a un mayor riesgo de prematuridad. Las sustancias que producen las bacterias pueden debilitar las membranas que rodean al saco amniótico y provocar su ruptura temprana. Incluso si las membranas permanecen intactas, las bacterias pueden causar una infección e inflamación en el útero, lo que podría acabar provocando un parto prematuro (Guerrero Sausa, et al., 2016).

En el año 2018 Yim y colaboradores publicaron los resultados de una revisión sistemática y un metaanálisis que relaciona el uso de corticoides prenatales con ROP. La administración de corticoides prenatales fue asociada con un menor riesgo de desarrollo de ROP (OR no ajustado $=0.82,95 \% \mathrm{Cl} 0.68$ to 0.98 ; OR ajustado $=0.67$, 
$95 \% \mathrm{Cl} 0.47$ to 0.94 ) y progresión a ROP severa (OR no ajustado $=0.58,95 \% \mathrm{Cl}$ 0.40 to 0.86 ) (Galina, 2018).

\section{Conclusión}

Luego de la recopilación de información se determinó que los factores de riesgo, más frecuentes y con asociación estadística a Retinopatía del prematuro fueron los factores biológicos, obstétricos, patológicos y psicosociales, se identificó a la edad materna (mujeres en edades muy jóvenes o muy avanzadas) como un factor de riesgo biológico indirecto para retinopatía del prematuro, al tabaquismo (ya sea consumo de nicotina o nornicotina) como un factor de riesgo psicosocial para el desarrollo de retinopatía del prematuro, dentro de los factores de riesgo patológicos se identificó a la hipertensión durante el embarazo, la diabetes mellitus, infección del tracto urinario, anemia por deficiencia de hierro y el aumento del recuento de glóbulos blancos y dentro factores de riesgo obstétricos se encontró edad gestacional, restricción del crecimiento intrauterino, ruptura de membranas, vía de resolución del parto, concepción asistida y corioamnionitis.

\section{Declaración}

El estudio se realizó con fines médicos, de acuerdo al Código de Ética y Buenas Prácticas COPE.

\section{Conflicto de intereses}

La autora declara no tener ningún conflicto de intereses.

\section{Referencias}

Camba Longueira, F., Perapoch López, J. \& Martín, B. (2008). Retinopatía del prematuro. In Protocolos de Neonatología. 2 ed. España, AEP, 443-447. https://www. aeped.es/sites/default/files/documentos/46.pdf 
Cauich, L. A. (2017). Caracterización epidemiológica de la retinopatía del prematuro en el Hospital de la Amistad Corea-México. Período 2005 a 2014. Perineonatología y reproduccion humana. 1(1). doi: https://doi.org/10.1016/j.rprh.2017.10.001.

Dai, A. I. (2015). Anemia maternal de la deficiencia de hierro como factor de riesgo para el desarrollo de la retinopatía de la precocidad. Neurología pediátrica, 53(2), 146 - 150. doi:https://doi.org/10.1016/j.pediatrneurol.2015.04.002.

Darlow, B., Hutchinson, J., Henderson-Smart, D., Donoghue, D., Simpson, J., \& Evans, N. (2005). Prenatal risk factors for severe retinopathy of prematurity among very preterm infants of the Australian and New Zealand neonatal network. Pediatrics 115(4), 990-996. https://doi.org/10.1542/peds.2004-1309

Guerrero Sausa, M., Sánchez Saavedra, S., \& Maguiña, L. D. (2016). Factores de riesgo que inciden en la retinopatía del prematuro en el servicio de neonatología en un hospital del ministerio de salud (en línea). Revista Científica Salud \& Vida Sipanense,1, 6-19. http://revistas.uss.edu.pe/index.php/SVS/article/view/359/347

Kim, S. J. (2018). Retinopathy of prematurity: a review of risk factors and their clinical significance (en línea). Survey of Ophthalmology, 618 - 637. doi:https://doi.org/10.1016/j.survophthal.2018.04.002.

Rivera-Rueda, M., Fernández-Carrocera, L., Salgado-Valladares, M., Cordero-González, G., Coronado-Zarco, I., \& Cardona-Pérez, J. (2020). Análisis de la frecuencia y factores de riesgo asociados a la retinopatía del prematuro en neonatos de muy bajo peso al nacer. Boletín Médico del Hospital Infantil de México 77(3), 135 - 141. https://doi.org/10.24875/BMHIM.20000203

Woo, S. P. (2012). Efectos de la inflamación maternal y placentaria sobre retinopatía de la precocidad. Graefes Arch Clin Exp Ophthalmol. 250, 915-923. doi:https:// doi.org/10.1007/s00417-011-1648-2

Yau, G., Lee, J., Tam, V., Yip, S., Cheng, E., Liu, C., . . Wong, I. (2015). Incidence and risk factors for retinopathy of prematurity in multiple gestations: a Chinese population study (en línea). Medicine. https://doi.org/10.1097/MD.0000000000000867 
Zhang, Y. M. (2015). Nornicotine and nicotine induced neovascularization via increased VEGF/PEDF ratio (en línea). Ophthalmic Research 55(1), 1-9. doi:https://doi.org/10.1159/000440847

\section{Sobre la autora \\ Nulvi Stefanni Portillo sosa}

Es estudiante de la carrera médico y cirujano autora de la investigación "Eficacia del shampoo de bebe vs carboximetilcisteina en ojo seco" y de la investigación "Eficacia de hoja de eucalipto en las principales cepas que producen neumonía". Carrera medico y cirujano, centro universitario de oriente CUNORI, Universidad de San Carlos de Guatemala.

Copyright (c) Nulvi Stefanni Portillo sosa

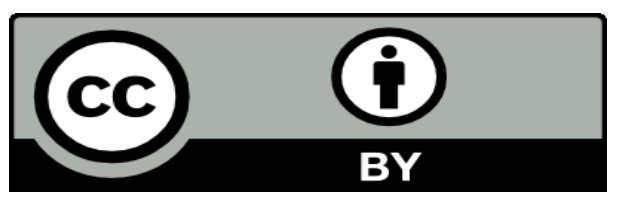

Este texto está protegido por una licencia CreativeCommons 4.0.

Usted es libre para compartir, copiar y redistribuir el material en cualquier medio o formato y adaptar el documento, remezclar, transformar y crear a partir del material para cualquier propósito, incluso comercialmente, siempre que cumpla la condición de atribución: usted debe reconocer el crédito de una obra de manera adecuada, proporcionar un enlace a la licencia, e indicar si se han realizado cambios. Puede hacerlo en cualquier forma razonable, pero no de forma tal que sugiera que tiene el apoyo del licenciante o lo recibe por el uso que hace. 